\title{
Perinatal Carcinogenesis
}

National Cancer Institute

\section{Source}

National Cancer Institute. Perinatal Carcinogenesis. NCI Thesaurus. Code C19941.

Studies of the mechanisms by which maternal exposure to carcinogens cause cancer in in children. 